\title{
Plasmonic Properties of Vertically Aligned Nanowire Arrays
}

\author{
Hua Qi, O. J. Glembocki, and S. M. Prokes \\ Electronics Science and Technology Division, US Naval Research Laboratory, Washington, DC 20375, USA \\ Correspondence should be addressed to Hua Qi, qhqihua@yahoo.com
}

Received 13 July 2011; Revised 20 September 2011; Accepted 4 October 2011

Academic Editor: Steve Acquah

Copyright (C) 2012 Hua Qi et al. This is an open access article distributed under the Creative Commons Attribution License, which permits unrestricted use, distribution, and reproduction in any medium, provided the original work is properly cited.

Nanowires (NWs)/Ag sheath composites were produced to investigate plasmonic coupling between vertically aligned NWs for surface-enhanced Raman scattering (SERS) applications. In this investigation, two types of vertical NW arrays were studied; those of $\mathrm{ZnO}$ NWs grown on nanosphere lithography patterned sapphire substrate via vapor-liquid-solid (VLS) mechanism and Si NW arrays produced by wet chemical etching. Both types of vertical NW arrays were coated with a thin layer of silver by electroless silver plating for SERS enhancement studies. The experimental results show extremely strong SERS signals due to plasmonic coupling between the NWs, which was verified by COMSOL electric field simulations. We also compared the SERS enhancement intensity of aligned and random $\mathrm{ZnO}$ NWs, indicating that the aligned NWs show much stronger and repeatable SERS signal than those grown in nonaligned geometries.

\section{Introduction}

Nanostructures, especially nanowires (NWs), have inspired much interest due to their potential applications in electronics, photonics, and life science [1-5]. In particular, NWs used for surface-enhanced Raman scattering (SERS) are of significant interest since SERS spectra can provide trace level detection and a chemical fingerprint by which a molecule can be identified. Several NWs types have been used to investigate the SERS effect, including silver NWs $[6,7]$, gold NWs [8], semiconductor NWs [9], and dielectric NWs [10-12]. It has been demonstrated that the geometry of the nanowires and the orientation of the molecules play an important role in the SERS process [10-14]. However, most of the reported NW structures were randomly distributed on a surface, where the largest SERS enhancements noted were in the NW crossing [10] and between the NW and the substrate [14]. Since it has been shown experimentally that the largest enhancements were between two closely aligned parallel NWs [13], an obvious extension of this result is to investigate the SERS enhancements of a large number of aligned parallel NW arrays. Thus, in this work, we have investigated the effect of close parallel NW alignment on the SERS response and on the reproducibility of the SERS signal. In addition, we have also investigated the plasmonic properties of periodic and nonperiodic NW arrays.
Since the "hot spots" or regions of plasmon coupling are the region from which SERS would have the largest enhancement and closely aligned NW should experience coupling along the whole lengths of the wires, we have produced arrays of plasmonic nanowires to investigate this SERS effect. Here we used two types of vertical NWs, ZnO NWs, and Si NWs, respectively, to investigate SERS enhancement due to the plasmonic coupling between closely packed NWs. Many lithographic techniques have been used to fabricate ordered patterns on a surface, including photolithography, e-beam lithography $[15,16]$, ion beam lithography [17], $\mathrm{X}$-ray lithography $[18,19]$, nanoimprint lithography [20, $21]$, and scanning probe lithography [22, 23]. However, all of these techniques are time consuming and costly. Here we employed the nanosphere lithography (NSL) approach [2426], which has the advantages of low cost and simplicity, to produce the hexagonal gold dot patterns on c-sapphire and to successfully grow vertically aligned $\mathrm{ZnO}$ nanowires by the well-known VLS process. In this way, the $\mathrm{ZnO}$ NWs can be arranged in a repeatable hexagonal pattern creating ordered and aligned NW arrays for surface-enhanced Raman scattering (SERS) applications. The closely packed vertical Si NWs were fabricated by wet chemical etching [27]. The Si NWs and $\mathrm{ZnO} \mathrm{NWs}$ arrays were coated with a thin layer of $\mathrm{Ag}$, using an electroless plating technique, and their plasmonic properties were investigated. 


\section{Experimental Details}

A bare c-sapphire piece with the size of $1 \times 1 \mathrm{~cm}^{-1}$ was cleaned in a piranha solution at $90^{\circ} \mathrm{C}$ for 30 min to produce a hydrophilic surface, rinsed by DI- $\mathrm{H}_{2} \mathrm{O}$, and dried under a flow of nitrogen. Commercial silica nanospheres (NSs) slurry, with a diameter of $300 \mathrm{~nm}$, was further diluted in a mixture of the surfactant Triton X-100 and methanol ( $1: 400$ by volume), with a dilution fraction of $1: 3$. NS lithography was performed on the sapphire surface by spin-coating the diluted silica nanosphere at a speed of $3600 \mathrm{rpm}$ for $60 \mathrm{~s}$. The sapphire surface, having a single layer of closely packed NS, was then covered by $10 \mathrm{nms}$ of gold, using an FC-2000 Temescal e-beam metal evaporation system, at a rate of $0.5 \AA / s$. After the gold deposition, the NS were removed by sonicating in DI- $\mathrm{H}_{2} \mathrm{O}$ for 1-2 minutes. Well-ordered gold particles in hexagonal patterns were left on the sapphire surface, and they served as a catalyst during the vertical $\mathrm{ZnO}$ growth.

Using the sapphire substrate prepared above, the growth of the vertical $\mathrm{ZnO}$ NWs was carried out in a horizontal furnace via the vapor-liquid-solid (VLS) growth mechanism. The mixture powder of $\mathrm{ZnO}: \mathrm{C}$ ( $1: 1$ by weight) used as the source was placed at one end of an alumina boat, and the substrate with the gold pattern was put directly above the material sources, face down. The furnace was heated to $900^{\circ} \mathrm{C}$ while flowing simultaneously a mixture of argon and oxygen gases in a ratio of $25: 1$ through the tube.

For the formation of the Si NW arrays, commercial p-type Si (100) wafer with narrow resistivity range of 6.3$7.0 \Omega-\mathrm{cm}$ was cleaned by piranha solution for $30 \mathrm{~min}$ at $90^{\circ} \mathrm{C}$ and then rinsed by DI water. Then $5 \% \mathrm{HF}$ aqueous solution was used to remove the oxide layer for $2 \mathrm{~min}$, which generated a fresh Si surface passivated with $\mathrm{H}$-termination. The resulting clean Si wafer was put into a mixture of $4.8 \mathrm{M}$ $\mathrm{HF}$ and $0.005 \mathrm{M} \mathrm{AgNO}_{3}$ solution for $1 \mathrm{~min}$. Then the wafer with a layer of silver was taken out of the solution, rinsed with DI water, and immediately immersed into wet etching chemicals of $4.8 \mathrm{M} \mathrm{HF}$ and $0.4 \mathrm{M} \mathrm{H}_{2} \mathrm{O}_{2}$ in the dark. After $30 \mathrm{~min}$, the wafer was taken out, rinsed with DI water, and immersed in dilute $\mathrm{HNO}_{3}(1: 1 \mathrm{v} / \mathrm{v})$ to dissolve the extra Ag catalyst. Finally the wafer was washed with $5 \% \mathrm{HF}$ again to remove the oxide layer, cleaned with DI water, and dried under a flow of $\mathrm{N}_{2}$. All was carried out at room temperature.

An electroless silver plating approach was employed to produce $\mathrm{ZnO}$ or Si NWs core/Ag sheath composites for the SERS investigations. The detailed procedure has been well demonstrated in our previous report [11, 12]. Briefly silver mirror reaction was used to produce neutral Ag in solution, and the freshly formed Ag nanoparticles were simultaneously deposited on NW surface, coating the whole surface of the nanowires.

A LEO scanning electron microscope (SEM) with energy dispersive X-ray (EDX) was used to investigate the topographies of NS patterned surface, growth of the vertical $\mathrm{ZnO}$ NWs, etching of the Si NWs, and electroless silver plating of the vertical $\mathrm{ZnO}$ or Si NWs. The SERS measurements of $\mathrm{ZnO}$ NWs were carried out utilizing a well-known SERS molecule, benzene thiol (BT), and a confocal $\mu$-Raman system which consisted of a Mitutoyo Microscope and an Ocean Optics QE65000 spectrometer equipped with a thermoelectrically cooled CCD. The $514.5 \mathrm{~nm}$ line of an Ar ion laser was used as the excitation source. The microscope utilized a 100x 0.7 NA objective for focusing the laser light and was coupled to the spectrometer through a fiber optic cable. The spectra were collected with low laser power of $0.75 \mathrm{~mW}$ at the sample. This was done to prevent desorption and damage to the SERSactive molecules, benzene thiol, and to prevent alterations to the Ag layer. The SERS behavior of Si NWs arrays was performed using a Delta Nu system which consists of an Olympus Microscope and a Raman spectrometer equipped with a thermoelectrically cooled CCD. The $785 \mathrm{~nm}$ line of Ti: Sapphire laser was used as the excitation source to detect the SERS strength dependence on a single NW/silver composite. The microscope utilized a 50x 0.75 NA objective for focusing the laser light. The spectra were collected with a laser power of $3 \mathrm{~mW}$ at the sample.

\section{Results and Discussion}

A single layer of closely packed silica nanospheres produced by self-assembly is shown in Figures 1(a) and 1(b). By this simple and high throughput way, the NS patterned area tens of microns in size can be easily produced. After the deposition of $10 \mathrm{~nm}$ of gold, the liftoff was performed in DI- $\mathrm{H}_{2} \mathrm{O}$ by sonicating. Hexagonal periodic Au particle arrays were obtained, as shown in Figure 1(c). When performing the sonicating lift-off process, 1-2 minutes was used. Longer sonication time usually led to the loss of the hexagonal gold patterns, while a shorter time resulted in incomplete liftoff of the nano-spheres from the substrate. Figure 1(d) shows an SEM image after the patterned sample was lifted off and annealed at $500^{\circ} \mathrm{C}$ for $10 \mathrm{~min}$. The distance between two nearest nanoparticle centers is around $170 \mathrm{~nm}$. Considering the $3 \mathrm{D}$ structure of the gold particles, the spacing may even be smaller, down to less than $100 \mathrm{~nm}$, which provides one way to obtain ordered $\mathrm{ZnO} \mathrm{NW}$ arrays for further plasmonic studies.

For the vertical $\mathrm{ZnO}$ nanowire growth, the well-known vapor-liquid-solid (VLS) growth mechanism was used. Due to the need to form a eutectic with the $\mathrm{Au}$ dots for NW growth, high selectivity of the growth resulted, and well-ordered $\mathrm{ZnO} \mathrm{NW}$ arrays were obtained, as shown in Figure 2(a). Since the NW growth rate depends on the size of the initial Au catalyst, some differences in the NW total lengths were noted. However, the hexagonal patterns are quite recognizable. To produce the patterned nanowire arrays required, we optimized the growth time to 10 minutes in order to control the nanowire growth length, as shown in Figure 2(a). In the case of longer NWs, the detailed information is discussed in what follows. In addition, the distance between the gold catalyst can be controlled by varying the size of the nanospheres, which provides a way to control the spacing between the vertical NWs. A representative image of the vertical $\mathrm{ZnO} \mathrm{NW}$ covered with a thin layer of silver using the electroless plating approach is shown in Figure 2(b), by which the roughness of the silvercoated NW surface is visible. 


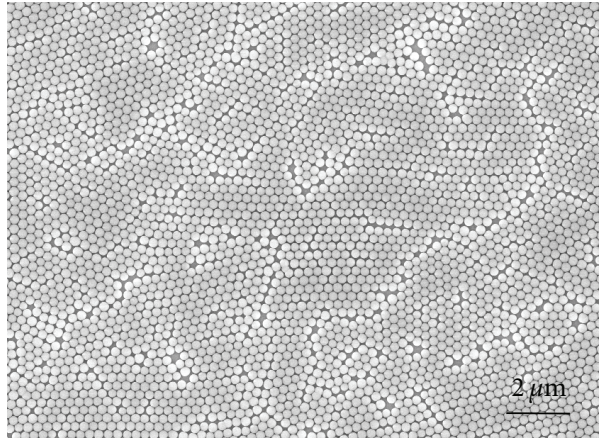

(a)

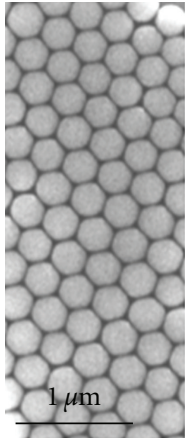

(b)



(c)

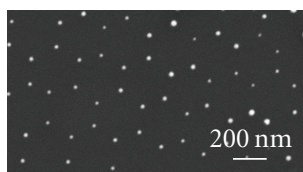

(d)

FIgURE 1: SEM images of monolayer of $300 \mathrm{~nm}$ silica nanospheres (a, b), hexagonal patterns after liftoff by sonicating (c, d).

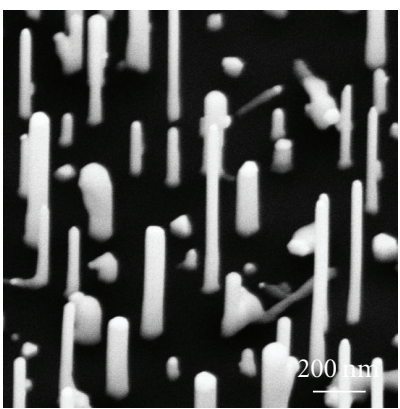

(a)

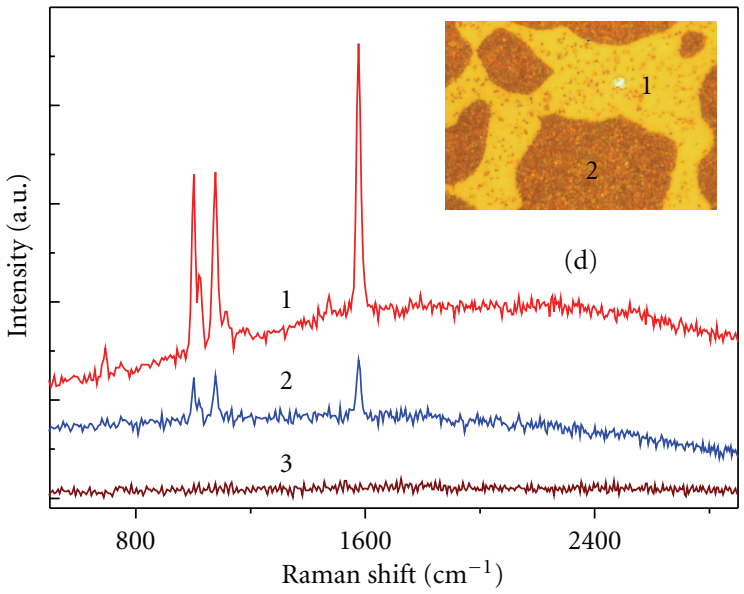

(c)

Figure 2: SEM images of short $\mathrm{ZnO}$ NWs as grown (a) and after silver coating on ZnO NWs surface (b); surface-enhanced Raman spectra (c) on silver-coated NWs at patterned area (red line 1), random area (blue line 2), and no NWs area (brown line 3 ) microscope image of SERS measurement sample (d).

Representative surface-enhanced Raman scattering (SERS) spectra of three regions are shown in Figure 2(c), including a well-ordered NWs area, random NWs area, and no NWs area. Figure $2(\mathrm{~d})$ is a corresponding optical microscope image, showing the different areas. The major Raman peaks at 1002, 1071, and $1576 \mathrm{~cm}^{-1}$ can, respectively,

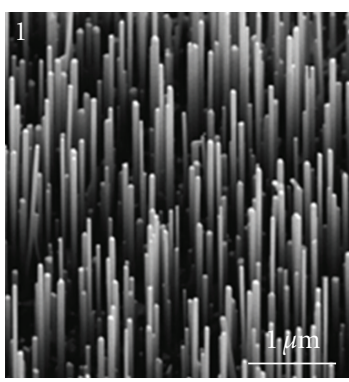

(a)

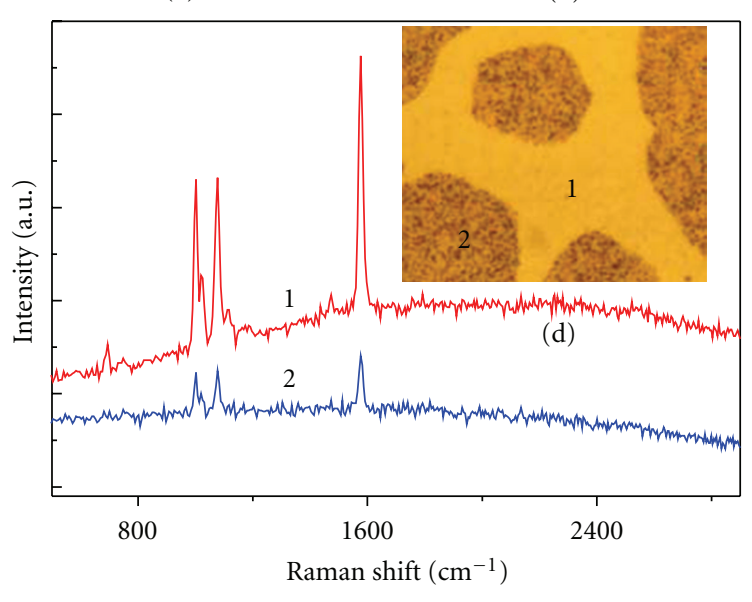

(c)

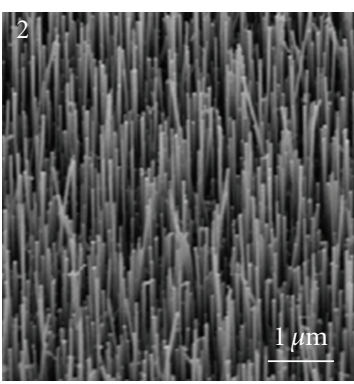

(b)
Figure 3: SEM images of $\mathrm{ZnO}$ NWs as grown (a) pattern area and (b) random area; surface-enhanced Raman spectra (c); (d) microscope image of SERS measurements of area 1 and 2.

be assigned to symmetric ring breathing, in plane $\mathrm{C}-\mathrm{H}$ bending and in plane $\mathrm{C}-\mathrm{C}$ stretching of the phenyl ring from the active molecules, benzene thiol (BT), which are in good agreement with those reported previously [28-33]. It is obvious that the strongest SERS signal is obtained on the wellordered vertical NWs arrays area, while no enhancement is received on the area without NWs, further confirming the important effect of dielectric ZnO NW on SERS mechanism, as reported previously [10]. The intensity of the $1567 \mathrm{~cm}^{-1}$ 


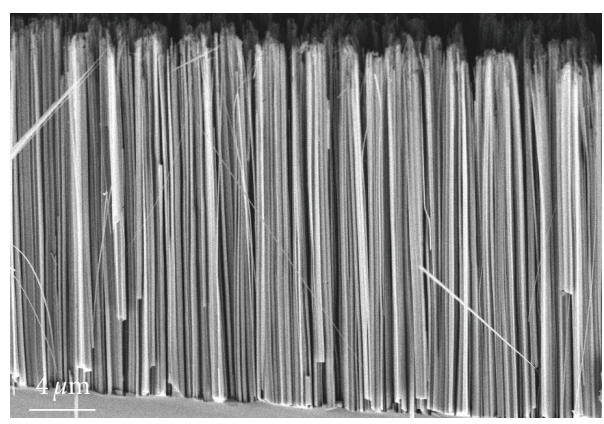

(a)

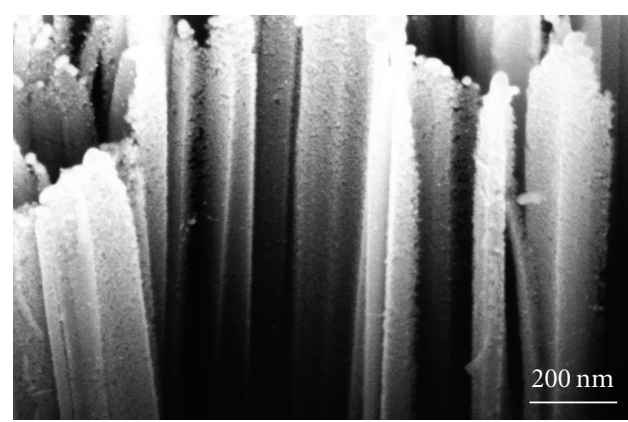

(b)

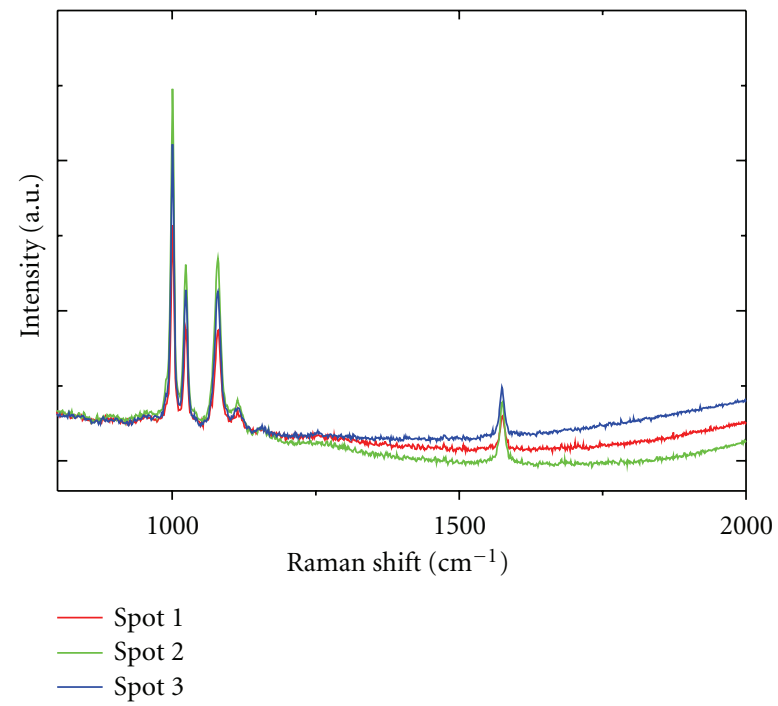

(c)

FIGURE 4: Representative SEM images of Si NWs produced by wet chemical etching (a), and silver-coated Si NWs (b). The sample stage was tilted $45^{\circ}$ when imaging. (c) Surface-enhanced Raman spectroscopy (SERS) patterns at different spots.

SERS band on the patterned vertical NW area is about 4 times stronger than those of the random NW area. This observation can be attributed to the reproducibility of the wellpatterned NWs, while the NWs on the random area are stochastic. It has been demonstrated that the randomly distributed dielectric NW core/Ag sheath composite lying on the surface can also produce notable SERS signal due to the interactions between silver-silver particles and silver-NW substrate $[11,12]$. In addition, there may be plasmonic coupling in the well-ordered area because of the close distance between the adjacent aligned NWs. Since this effect increases with the number of aligned NWs, this may also be contributing to the enhanced SERS measured. It is well known that the SERS effect relies on enhanced electric fields that are created because of light-induced plasmons in the silver nanoparticles. As reported earlier [11], the plasmons in closely spaced metal nanostructures are coupled to form regions of very high field, further enhancing the SERS response.

The strongest SERS enhancement displayed above was obtained using the short vertical $\mathrm{ZnO}$ NWs with the length of less than $700 \mathrm{~nm}$. In fact, we also investigated the SERS behavior on the well-ordered and patterned sample with longer NWs. Several-microns-long vertical $\mathrm{ZnO} \mathrm{NWs}$ of patterned area and unpatterned area are, respectively, shown in Figures 3(a) and 3(b). As shown in Figure 3(c), the SERS intensity is moderately greater in the aligned case than in the nonaligned case. Figure 3(d) is an optical microscope image of the measured surface area with ordered or random $\mathrm{ZnO}$ NWs. It is well known that SERS signals depend on density, size, morphology, and thickness of the coated material. However, in the case of closely packed and vertically aligned random NWs, the silver particle coverage is gradually decreasing as a function of depth, from the NWs top to the bottom, which can be attributed to the well-known capillary effect since we introduced solutions to perform the electroless silver plating. This defect of the silver particles coverage on NWs could affect the strength of SERS due to lack of "hot spots." In fact, the NW density of unpatterned area is greater than in the patterned case. This is logical, since in the unpatterned area, a continuous Au film is used as a catalyst, and thus, in the VLS growth, a large number of catalyst islands form to grow NWs. In the patterned area, however, only a very small fraction of the substrate is covered in $\mathrm{Au}$ islands, since most of the area is taken up by the nanospheres, which of course get removed prior to growth, leaving only the small triangular regions of $\mathrm{Au}$, as shown in Figure 1(c). 


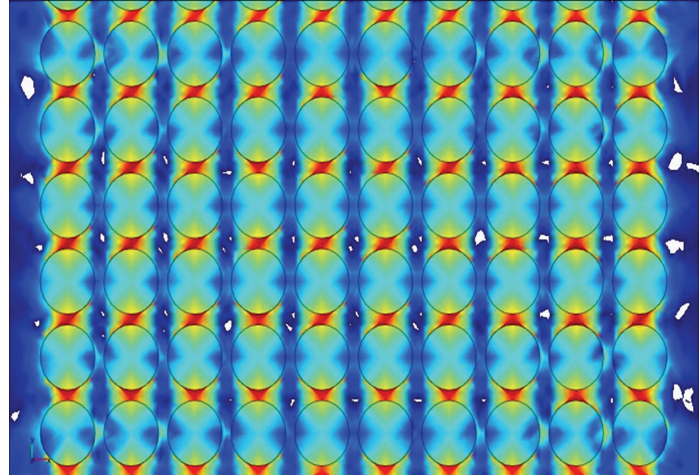

(a) Top view

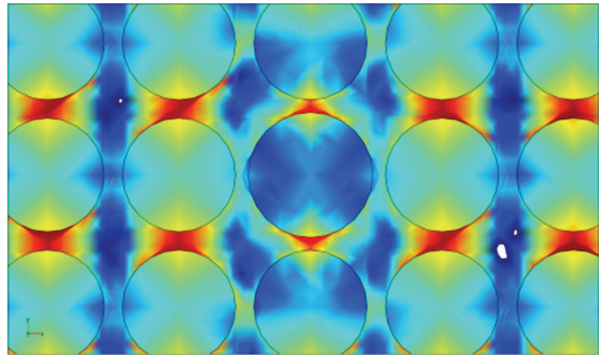

(c) $10 \%$ wider wire



(b) Side view

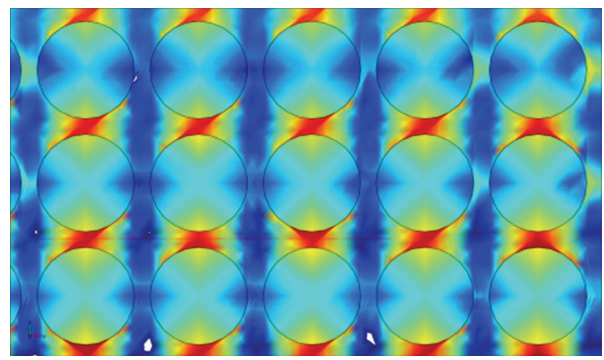

(d) 2 rows above defect

FIGURE 5: COMSOL simulations of the plasmonic coupling of parallel Si NWs; (a) top view; (b) side view, (c) local enhacement decrease (less intense red regions) with larger NW diameter "defect" and (d) no effect on SERS enhancement of the NW arrays two rows away from NW "defect."

Since, as noted above, it is harder to get a high coverage of the silver particles along the whole length of the NW in closely spaced random NW arrays due to the capillary effect, one might expect a reduction in the SERS response, although a higher totally NW density is present. In the case of the patterned area, the NWs result in better Ag coverage due to the lower NW density and thus a more open structure. It is also noted that increasing the plating time or concentration of the plating solution does not help to improve the silver plating of closely packed NWs and usually results in NW breakage or a pileup of silver nanostructures on the tip of the NWs, blocking the way to further silver plating of the NWs parting depth.

It is hard to investigate the detailed morphology of the nanostructures at high-resolution SEM due to the charging issue of sapphire substrate, on which vertical $\mathrm{ZnO}$ nanowires were usually grown. Thus, we employed semiconductor Si NWs, which was produced by chemical wet etching and has similar vertically aligned nanostructures to $\mathrm{ZnO}$ NWs. A typical side view SEM image of Si NWs is shown in Figure 4(a), indicating that the NWs are uniform with a high density and close spacing. The lengths of the NWs are close to $30 \mu \mathrm{m}$. As shown in Figure 4(b), the vertical Si NWs were covered by a thin layer of silver nanoparticles by electroless plating as described above. The SERS measurements at different spots were performed. As shown in Figure 4(c), the SERS intensity is very uniform across Si NWs array sample, demonstrating that the wet chemical etching and electroless plating results in repeatable and uniform plasmonic NW large area arrays. The lines for the SERS-active molecules, BT, are very similar to those reported previously [28-33]. When the SERS intensity of the Si NW arrays was compared to the $\mathrm{ZnO} \mathrm{NW}$ arrays using the $514 \mathrm{~nm}$ laser line, an order of magnitude greater signal was noted for the Si NWs. This is most likely to a much higher density of wires, which are also more closely spaced, leading to greater plasmon coupling.

Vertical NWs with smaller separation distance can result in the formation of hot spots corresponding to very intense electric field. Kottmann and Martin [34] have shown that coupled parallel metallic wires lead to a significantly enhanced coupled plasmon resonance in the overall structure. Finite element COMSOL simulation of the NW array plasmonic properties is shown in Figure 5. As can be seen in Figure 5(a), parallel and closely packed NWs/Ag composites induced high electric field along the whole length of the nanowires, which result in the very large enhancement of the observed SERS signals. Figure 5(b) is a side view of the parallel NW/Ag composites, clearly demonstrating the strong plasmonic coupling between the NW/Ag composites. Furthermore, on average, the strong plasmonic coupling is not dramatically influenced by small variations in the nanowire diameters. Figure 5(c) shows the influence of a $10 \%$ increase in the thickness of the central nanowire. The local fields around the nanowire are reduced leading to a factor of 4 decrease in the very local SERS enhancement. However, note that areas that are only several nanowires away from our defect show virtually no change in the SERS enhancement. In fact as shown in Figure 5(d), the line of nanowires that 
are two rows above the defect are not impacted by the defect at all. Thus, the plasmonic fields behave similarly to semiconductor bands that have local densities of states from impurities that are isolated from the semiconductor conduction or valence bands. This analogy further suggests that even in the "amorphous" case, which is random distributions of aligned nanowires, there should still be a high SERS uniformity across the array, as shown in Figure 4.

\section{Conclusion}

We have successfully produced ordered, parallel $\mathrm{ZnO}$ and $\mathrm{Si}$ nanowire arrays on c-sapphire and Si substrates, respectively. The area of periodic ZnO NWs exhibits 4 times stronger SERS signal than those of random $\mathrm{ZnO}$ NWs, demonstrating the feasibility of using metal/dielectric nanowire periodic arrays to enhance the SERS signal. The wet chemically etched Si NWs were also studied, demonstrating the strong plasmonic coupling between the parallel NWs/Ag composites. These results are in good agreement with our COMSOL simulations of the closely packed vertical NWs/Ag composites. Since large SERS enhancements and SERS uniformity and repeatability are important issues, this study provides potential for the development of reliable, remotely deployable, and more sensitive optically based sensors.

\section{Acknowledgments}

We are thankful for the support of the Office of Naval Research (ONR), the American Society for Engineering Education program, the National Basic Research Program (2011CB933101) and Nanoscience Institute at Naval Research Lab (NRL).

\section{References}

[1] Y. Sun and J. A. Rogers, "Fabricating semiconductor nano/ microwires and transfer printing ordered arrays of them onto plastic substrates," Nano Letters, vol. 4, no. 10, pp. 1953-1959, 2004.

[2] T. Mårtensson, C. P. T. Svensson, B. A. Wacaser et al., "Epitaxial III-V nanowires on silicon," Nano Letters, vol. 4, no. 10, pp. 1987-1990, 2004.

[3] P. Mohan, J. Motohisa, and T. Fukui, "Controlled growth of highly uniform, axial/radial direction-defined, individually addressable InP nanowire arrays," Nanotechnology, vol. 16, no. 12, pp. 2903-2907, 2005.

[4] S. D. Hersee, X. Sun, and X. Wang, "The controlled growth of GaN nanowires," Nano Letters, vol. 6, no. 8, pp. 1808-1811, 2006.

[5] M. T. Björk, C. Thelander, A. E. Hansen et al., "Few-electron quantum dots in nanowires," Nano Letters, vol. 4, no. 9, pp. 1621-1625, 2004.

[6] R. Gunawidjaja, S. Peleshanko, H. Ko, and V. V. Tsukruk, "Bimetallic nanocobs: decorating silver nanowires with gold nanoparticles," Advanced Materials, vol. 20, no. 8, pp. 15441549, 2008.

[7] S. J. Seung, J. M. Baik, and M. Moskovits, "Polarizationdependent surface-enhanced raman scattering from a silvernanoparticle-decorated single silver nanowire," Nano Letters, vol. 8, no. 10, pp. 3244-3247, 2008.
[8] H. Wei, F. Hao, Y. Huang, W. Wang, P. Nordlander, and H. Xu, "Polarization dependence of surface-enhanced Raman scattering in gold nanoparticle-nanowire systems," Nano Letters, vol. 8, no. 8, pp. 2497-2502, 2008.

[9] C. Fang, A. Agarwal, E. Widjaja et al., "Metallization of silicon nanowires and SERS response from a single metallized nanowire," Chemistry of Materials, vol. 21, no. 15, pp. 3542-3548, 2009.

[10] S. M. Prokes, O. J. Glembocki, R. W. Rendell, and M. G. Ancona, "Enhanced plasmon coupling in crossed dielectric/ metal nanowire composite geometries and applications to surface-enhanced Raman spectroscopy," Applied Physics Letters, vol. 90, no. 9, Article ID 093105, 2007.

[11] H. Qi, D. Alexson, O. Glembocki, and S. M. Prokes, "Plasmonic coupling on dielectric nanowire core-metal sheath composites," Nanotechnology, vol. 21, no. 8, Article ID 085705, 2010.

[12] H. Qi, D. Alexson, O. Glembocki, and S. M. Prokes, "The effect of size and size distribution on the oxidation kinetics and plasmonics of nanoscale Ag particles," Nanotechnology, vol. 21, no. 21, Article ID 215706, 2010.

[13] S. M. Prokes, D. A. Alexson, O. J. Glembocki, H. D. Park, and R. W. Rendell, "Effect of crossing geometry on the plasmonic behavior of dielectric core/metal sheath nanowires," Applied Physics Letters, vol. 94, no. 9, Article ID 093105, 2009.

[14] O. J. Glembocki, R. W. Rendell, S. M. Prokes, D. A. Alexson, A. $\mathrm{Fu}$, and M. A. Mastro, "Dielectric-Substrate interactions with metal/dielectric nanowires composites in surface-enhanced Raman spectroscopy," Physical Reviews B, vol. 80, no. 1, 2009.

[15] R. R. W. Pease, "Nanolithography and its prospects as a manufacturing technology," Journal of Vacuum Science \& Technology $B$, vol. 10, no. 1, pp. 278-285, 1992.

[16] M. A. McCord, "Electron beam lithography for $0.13 \mu \mathrm{m}$ manufacturing," Journal of Vacuum Science and Technology B, vol. 15, no. 6, pp. 2125-2129, 1997.

[17] J. Melngailis, A. A. Mondelli, I. L. Berry, and R. Mohondro, "A review of ion projection lithography," Journal of Vacuum Science and Technology B, vol. 16, no. 3, pp. 927-957, 1998.

[18] H. I. Smith and M. L. Schattenburg, "X-ray lithography, from 500 to $30 \mathrm{~nm}$ : X-ray nanolithography," IBM Journal of Research and Development, vol. 37, no. 3, pp. 319-329, 1993.

[19] J. P. Silverman, "X-ray lithography: status, challenges, and outlook for $0.13 \mu \mathrm{m}$," Journal of Vacuum Science and Technology B, vol. 15, no. 6, pp. 2117-2124, 1997.

[20] Y. S. Kim, K. Lee, J. S. Lee, G. Y. Jung, and W. B. Kim, "Nanoimprint lithography patterns with a vertically aligned nanoscale tubular carbon structure," Nanotechnology, vol. 19, no. 36, Article ID 365305, 2008.

[21] I. W. Moran, A. L. Briseno, S. Loser, and K. R. Carter, "Device fabrication by easy soft imprint nano-lithography," Chemistry Letters, vol. 20, no. 14, pp. 4595-4601, 2008.

[22] S. Manne, P. K. Hansma, J. Massie, V. B. Elings, and A. A. Gewirth, "Atomic-resolution electrochemistry with the atomic force microscope: copper deposition on gold," Science, vol. 251, no. 4990, pp. 183-186, 1991.

[23] W. T. Müller, D. L. Klein, T. Lee, J. Clarke, P. L. McEuen, and P. G. Schultz, "A Strategy for the chemical synthesis of nanostructures," Science, vol. 268, no. 5208, pp. 272-273, 1995.

[24] G. H. Chan, J. Zhao, E. M. Hicks, G. C. Schatz, and R. P. Van Duyne, "Plasmonic properties of copper nanoparticles fabricated by nanosphere lithography," Nano Letters, vol. 7, no. 7, pp. 1947-1952, 2007. 
[25] C. L. Haynes and R. P. Van Duyne, "Nanosphere lithography: a versatile nanofabrication tool for studies of size-dependent nanoparticle optics," Journal of Physical Chemistry B, vol. 105, no. 24, pp. 5599-5611, 2001.

[26] J. C. Hulteen, D. A. Treichel, M. T. Smith, M. L. Duval, T. R. Jensen, and R. P. Van Duyne, "Nanosphere lithography: sizetunable silver nanoparticle and surface cluster arrays," Journal of Physical Chemistry B, vol. 103, no. 19, pp. 3854-3863, 1999.

[27] M. L. Zhang, K. Q. Peng, X. Fan et al., "Preparation of largearea uniform silicon nanowires arrays through metal-assisted chemical etching," Journal of Physical Chemistry C, vol. 112, no. 12, pp. 4444-4450, 2008.

[28] C. Jiang, W. Y. Lio, and V. V. Tsukruk, "Surface enhanced raman scattering monitoring of chain alignment in freely suspended nanomembranes," Physical Review Letters, vol. 95, no. 11, Article ID 115503, pp. 1-4, 2005.

[29] J. Ding, V. I. Birss, and G. Liu, "Formation and properties of polystyrene-block-poly(2-cinnamoylethyl methacrylate) brushes studied by surface-enhanced raman scattering and transmission electron microscopy," Macromolecules, vol. 30, no. 5, pp. 1442-1448, 1997.

[30] W. M. Sears, J. L. Hunt, and J. R. Stevens, "Raman scattering from polymerizing styrene. I. Vibrational mode analysis," The Journal of Chemical Physics, vol. 75, no. 4, pp. 1589-1598, 1981.

[31] V. Zucolotto, M. Ferreira, M. Cordeiro et al., "Unusual interactions binding iron tetrasulfonated phthalocyanine and poly (allylamine hydrochloride) in layer-by-layer films," Journal of Physical Chemistry B, vol. 107, no. 16, pp. 3733-3737, 2003.

[32] R. Aroca and A. Thedchanamoorthy, "Vibrational studies of molecular organization in evaporated phthalocyanine thin solid films," Chemistry of Materials, vol. 7, no. 1, pp. 69-74, 1995.

[33] M. Lütt, M. R. Fitzsimmons, and D. Li, "X-ray reflectivity study of self-assembled thin films of macrocycles and macromolecules," Journal of Physical Chemistry B, vol. 102, no. 2, pp. 400-405, 1998.

[34] J. P. Kottmann and O. J. F. Martin, "Plasmon resonant coupling in metallic nanowires," Optics Express, vol. 8, no. 12, pp. 655-663, 2001. 

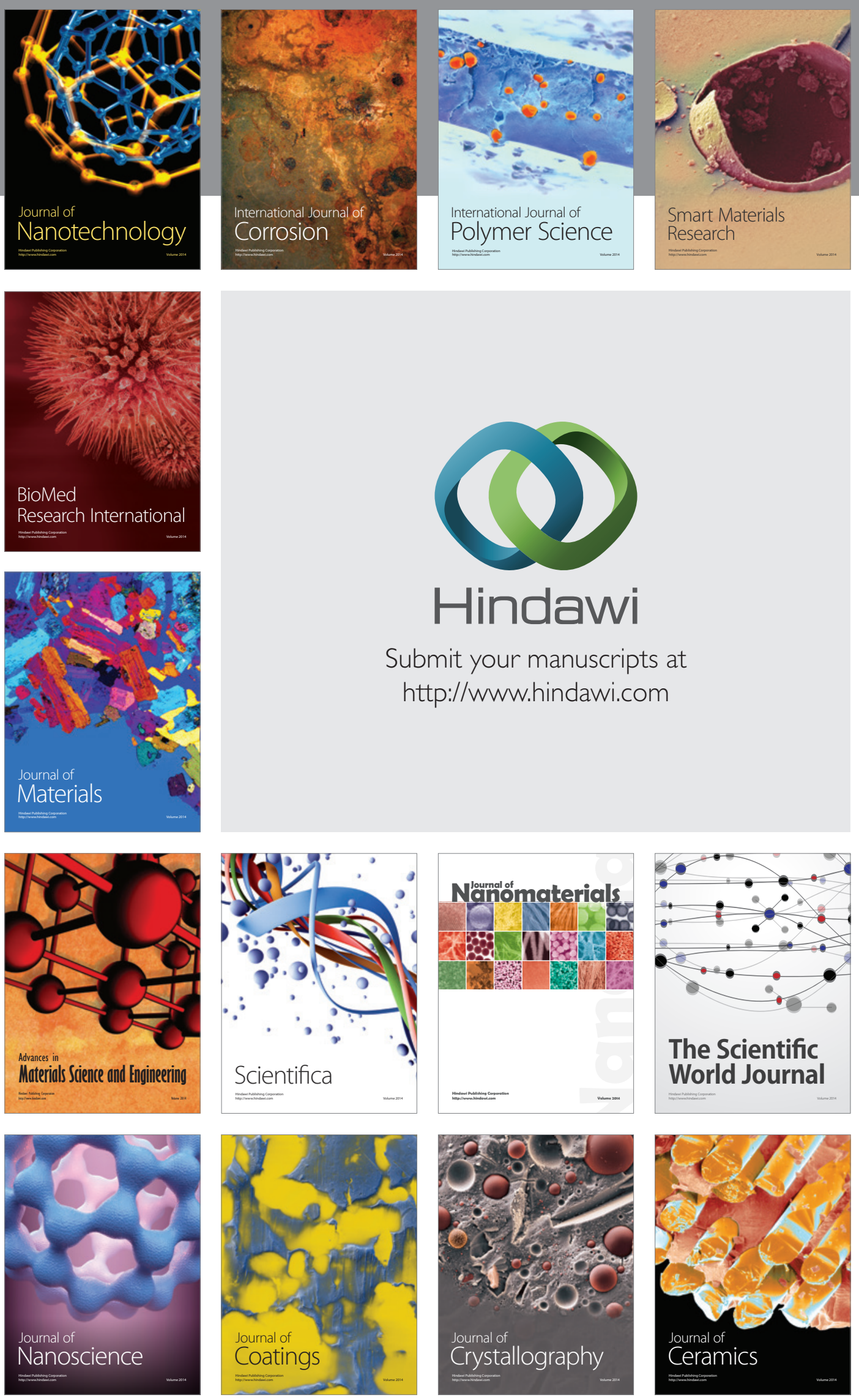

The Scientific World Journal

Submit your manuscripts at

http://www.hindawi.com

\section{World Journal}

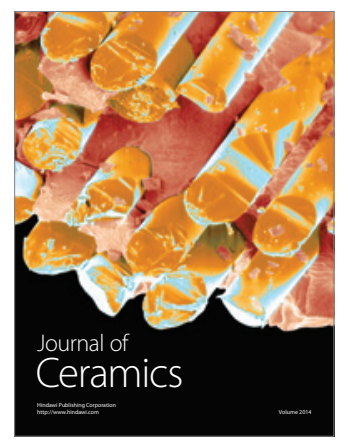

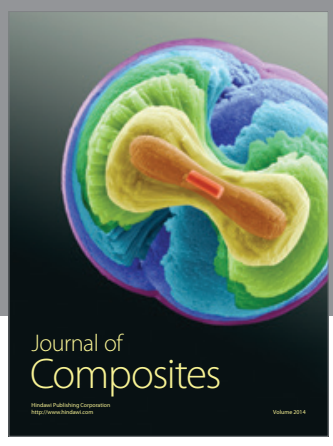
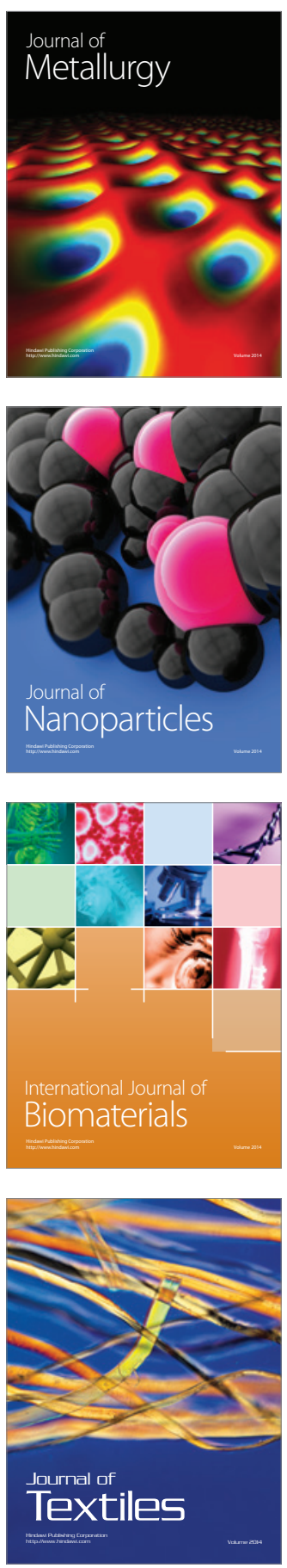